\title{
ENERGY SERVICES BRIDGING THE GAP BETWEEN RESIDENTIAL FLEXIBILITY AND ENERGY MARKETS
}

\author{
Ricardo André, Gisela Mendes, \\ Alexandre Neto, Pedro Castro \\ EDP NEW R.\&.D - Portugal \\ ricardo.andre@edp.pt
}

\author{
Andre Madureira, Jean Sumaili, \\ Clara Gouveia, Leonel Carvalho \\ INESC-TEC - Portugal \\ andre.g.madureira@inesctec.pt
}

\author{
Tuukka Rautiainen \\ EMPOWER - Finland \\ tuukka.rautiainen@empower.fi
}

\author{
Catherine Murphy-O'Connor \\ INDRA - Spain \\ cmurphy@indra.es
}

\author{
Andrea Michiorri, Alexis Bocquet, Alexis Gerossier \\ MINES-ParisTech, PERSEE \\ PSL - Research University - France \\ andrea.michiorri@mines-paristech.fr
}

\begin{abstract}
This article addresses the developments ongoing in SENSIBLE, an H2O2O funded project focused on energy storage and energy management, which demonstration occurs in Évora-Portugal, Nottingham-UK and Nuremberg-Germany. Currently presented work focus the concepts and developments necessary in order to make possible that residential clients can participate in a market environment with their electrical flexibility, also considering Distribution System Operator (DSO) needs when gird is under stress caused by any technical constraint. Moreover than the concept behind it is necessary to consider several developments: i) a low layer where residential assets will live in customers houses; ii) a high-level layers where market tools and DSO management tools will live; iii) an intermediate layer which bridge the gap between the low layer and high layer. These developments are a result of the ongoing works under one of SENSIBLE use cases which demonstrations occurs in a small village in Évora district in Portugal.
\end{abstract}

\section{INTRODUCTION}

Energy storage and management technologies at residential level coupled with distributed energy generation (DG) sources like PV can become a competitive and valuable source of energy flexibility which can be exploited by several energy players: i) residential clients - focused mostly on energy cost reduction; ii) Energy Service Providers (ESP) like retailers - focused on providing new energy services to end users but also in optimizing its market participation; iii) DSO focused on Quality-of-Service in dynamic distribution grids and iv) TSO focused in electrical system stability as a whole. This paper analyzes a case study which is being implemented under Project SENSIBLE ${ }^{1}$ where a high number of LV clients are equipped with an home energy management system (HEMS), storage devices (water heater, battery storage, or both), smart plugs and PV panels which due to its high capacity installed in demonstrator

1 H2020 -LCE08-14 - SENSIBLE Storage-Enabled Sustainable Energy for Buildings and Communities, Grant will create technical constraints in the grid for demonstration purposes. The demonstration work will test how this flexibility can be managed taking into account the different targets of the aforementioned energy players.

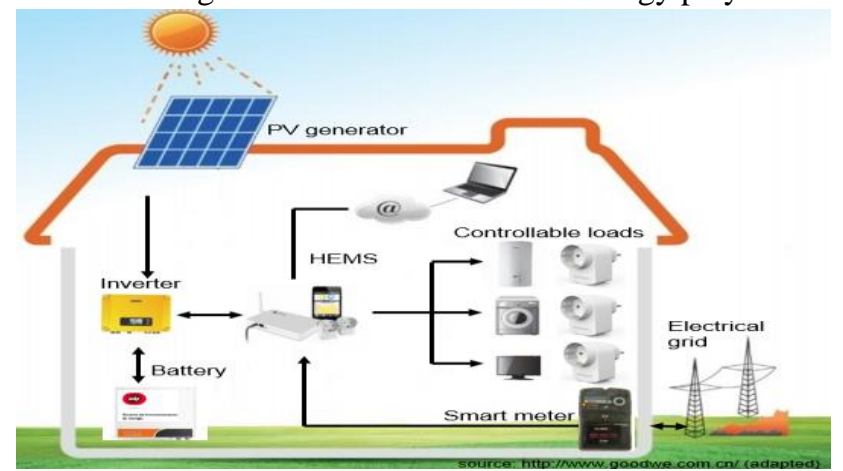

Figure 1 - Residential assets architecture

One hot topic in Europe embraces storage ownership: should it be a regulated asset or should it be a market asset, like a service provided to DSO. According to European regulation in case of a competitive environment occurs, this is the preferable approach. A critical aspect that should not be discarded is the stakeholders' engagement strategies in validation phase, since several equipment will be installed at customer's houses and where SENSIBLE has been a well-known case of success [4].

\section{CONCEPT AND APPROACH}

The case study takes place in Évora demonstrator of SENSIBLE, comprising 25 out of 240 clients connected to a LV rural grid. Each client will have installed the abovementioned residential equipment where the HEMS which will bridge the gap between the clients and energy markets through an Energy Service Provider.

Under the scope of this case study, each client will establish a contractual relationship with ESP that encompasses the energy supply and the flexibility contract that allows the ESP to manage the residential assets according to their real value in the market. The scheduling of flexibility that clients are willing to provide is set at the 
HEMS level, through a dedicated web interface with client.

Under SENSIBLE scope, flexibility may be valuable in a market environment, taking into account the multiple services for the flexibility buyers: i) minimize retailers' unbalance in market; ii) auxiliary service market; iii) in tertiary reserve market; iv) requested by DSO to solve grid technical constraints and maintain the quality of service standards. One of the biggest challenges is how should flexibility be valuated when it is critical to the DSO. In presented approach the market will drive the price and if this flexibility is necessary by the DSO the fair price is settled in a competitive approach.

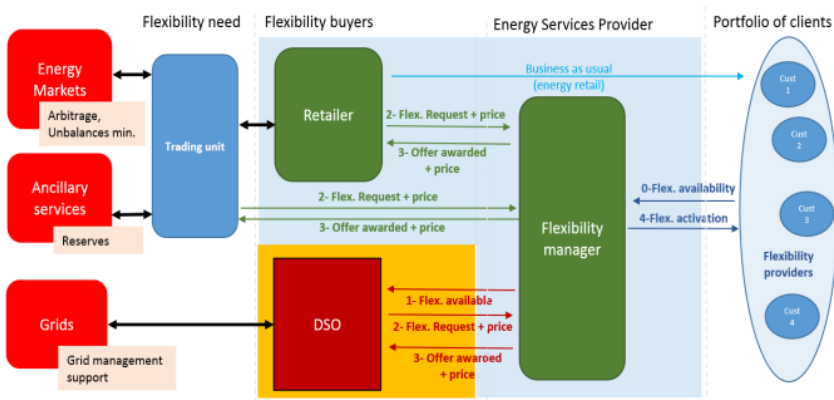

Figure 2 - Relation between actors

In summary the use case concept can be explained by the following sequence of actions, supposing that a retailer has an energy contract relationship in a business as usual approach with a portfolio of customers and an Energy Service Provider establish a flexibility contract with some of those clients. Moreover a day-ahead timeframe is considered for now and no technical constraints is foreseen. Otherwise DSO will have priority assessing this flexibility, which will be kept in step 1 .

1. The flexibility manager collects the available amount of flexibility from customers, depending on each customer availability. This info will be shared with the DSO which has priority accessing this flexibility for grid operation purposes. From retailer and trading unit perspective, flexibility value in market is already known since they are aware of the amount of unbalances and the trend of other markets like ancillary services markets.

2. The flexibility buyers (retailer, trading unit, DSO) will offer a price for that flexibility considering its value in market for market players and DSO will do the trade-off between two options: i) use this flexibility at that price; ii) use conventional DSO tools, like grid investment, own storage operation, grid reconfiguration, etc...

3. The ESP will offer that flexibility according the auction's result.

4. ESP will activate the flexibility for each customers agreed.

In an intraday timeframe two situations can occur: i) there is a market opportunity for retailer (in case of deviations) or trading unit (in case of energy markets or ancillary services market) and flexibility manager have a spread price in terms of day ahead case previously agreed; ii) the DSO detects a technical constraint and in such case it has priority purchasing that flexibility considering the market price reference.

\section{ARQUITECTURE AND TOOLS}

Error! Reference source not found. depicts the block diagram of the ICT architecture.

The RTP plays an important role bridging the gap between smart grid components and high level tools (such as analytics, forecast and market) which are needed to implement the use case. The RTP will also allow a real connection between clients (through HEMS) and retailers enabling a real market participation for residential sector through SENSIBLE energy management tools.

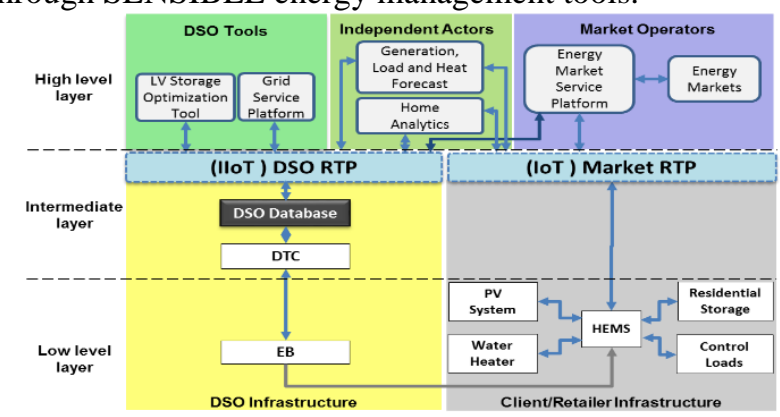

Figure 3 - ICT Architecture

The three main layers of the architecture are summarized below:

\section{Low level layer}

On the one hand we have the Client/Retailer infrastructure which enables the integration of residential/commercial clients in the operation of the distribution network. The main component of this infrastructure is the HEMS that is responsible for managing local resources in order to accomplish the minimization of power consumption and the maximization of the use of renewable energy through the management of storage. The flexibility resultant from the management of flexible resources such as storage and flexible loads (such as electric water heater) will be considered as an asset for enabling the participation of consumers in the operation of the system. This flexibility can provide valuable resources for DSO regarding the implementation of DSM schemes. The joint flexibility of the participating customers is computed and sent to the Market Platform via the Market RTP where the market participation of the clients is enabled.

The link between the client/retailer infrastructure and the DSO infrastructure is established through the HEMS taking into consideration the contracted power as well as information related to voltage and power measurements obtained from the Smart Meter (Energy Box - EB), while the later serves also as an interface gateway with the HEMS. The EB provides valuable data for the operation of LV systems characterizing the energy consumption and voltage in each node of the network.

\section{Intermediate layer:}

Within the DSO infrastructure, EDP Distribuição owns a smart grid infrastructure called InovGrid where Distribution Transformer Controller acts (not only) as a 
concentrator for Automatic Measuring Infrastructure (AMI) which will play a crucial role collecting information from the Energy Box (EB). The DTC concentrates the data collected by all the EB installed downstream and send it to the upstream central systems. The DSO Database represents the existing grid distribution management system, which encompasses the AMI application, the grid control and operation modules and the data collection and provision components. This component collects all the data communicated via DTC and acts as a middleware between the downstream smart grid (and all the grid connected devices) and the upstream Real Time Integration Platform (RTP).

The DSO RTP part of the Real Time Platform acts as the middleware communication infrastructure, collecting data from the downstream smart grid deployed infrastructure and making the data available to the higher level applications. In addition, it exchanges information between the high level tools as well as the commands provided by the DSO tools to the DSO Database.

The RTP can, in turn, be divided in two separated platforms: one operating in the Internet of Things (IoT) the Market RTP - and another in the Industrial Internet of Things (IIoT), the DSO RTP.

\section{High level layer:}

It is classified in three different blocks which are all connected to the RTP in order to collect data from the field or to send commands and setpoints downstream: i) DSO tools to detect grid constraints and compute the required amount of flexibility required; ii) The Energy Market Service Platform (EMSP) simulates the markets and determines the flexibility price; iii) Additionally, the independent actor tools provides load, generation and heat forecast and last but not least, the Home Analytics manage the granular flexibility from each house considering any restriction from clients and deliver it to the EMSP.

The DSO tools will be responsible for managing the distribution network, taking advantage of the several Distributed Energy Resources (DER) present, namely storage devices. In this case, a LV Optimization Tool is employed that aims at optimizing grid operation by using storage devices owned by the DSO, either located at the secondary substation level, either distributed in the LV grid. In addition, in case of constraints in the LV grid (for instance, voltage violations or branch overloads), flexibility from LV domestic clients (via the HEMS) may also be used in order to correct these technical problems. This flexibility is not meant to be included in any market mechanism and is to be used by the DSO only in case problems in grid operation occur and should always be ensured in order not compromise a safe operation of the LV grid.

The LV Optimization Tool is formulated as an Optimal Power Flow (OPF), developed specifically for LV grids, i.e. using a three-phase unbalanced representation of the LV grid due to the connection of single-phase units such as PV panels or loads. The tool will run both day-ahead (d1) in order to define the LV optimal strategy for the storage units and demand flexibility of customers and identify potential technical restrictions that cannot be solved by the algorithm. Then during the day (d), before sending the setpoints directly to the different resources, the tool will validate the set-points defined in the previous day based on the current state of LV network. This will avoid deteriorating the operation conditions when the forecasting error is high.

The Energy Market Service Platform (EMSP) is responsible for connecting the residential flexibility with the energy markets. Another role for the EMSP is to simulate the activities of a retailer willing to utilize flexibility. The platform consists of components that can handle metering data, customer information and manage energy portfolios. Furthermore, a flexible information exchange interface enables a smooth connection to other tools like the RTP. The use of flexibility in EMSP is determined in three steps.

1. Collect retailer related forecasting data to determine the day-ahead energy balance including available flexibility

2. Enable trading and auctioning between the retailer, DSO and residential client to determine an optimal use and valuation of flexibility

3. Negotiate the awarded flexibility to all the stakeholders in order to execute required control actions

First the EMSP collects consumption and production forecasts from the retailer's customers in order to determine the right allocation of flexibility. Using the forecasting data and prices from different markets the EMSP can calculate the value of residential flexibility for the retailer. In the second step, the EMSP receives flexibility requests from the DSO at grid node level in order to manage grid congestion. Likewise, the platform simulates retailer's actions and determines corresponding flexibility requests, including capacity and price, for the retailer. All the flexibility requests are compared on a 15minute basis to determine an optimal outcome. Finally, to guarantee end customers' position the EMSP also checks the technical costs of flexibility execution before accepting the outcome of the flexibility allocation. Last step in the sequence of the EMSP is to send the result of the trading/auctioning process to the retailer and the DSO in order to execute them. This phase is also established in 15minute intervals.

Day ahead and intraday operation can be enhanced by electric demand forecasts and micro generation forecast, especially when this depends on renewable resources, such as in the case of PV plants. In SENSIBLE, two forecast tools are developed:

\section{- Demand forecast \\ - Electric demand forecast \\ ○ Heating demand forecast \\ - $\quad$ PV production forecast}

Both the forecast tools provide both deterministic and probabilistic forecast, this means that, if $F_{t+h \mid t}$ denotes the 
cumulative distribution function and $q_{t+h \mid t}^{\alpha}$ the $\alpha$-quantile of the production $p_{t+h}$, forecast for the horizon $h$, conditionally to the information set $\mathrm{X}_{\mathrm{t}}$ available at instant $t$, we got:

$F_{t+h \mid t}(x)=\mathbb{P}\left(p_{t+h} \leq x \mid \mathrm{X}_{\mathrm{t}}\right)$ and $q_{t+h \mid t}^{\alpha}=F_{t+h \mid t}^{-1}(\alpha)$

The forecast are for the day ahead and have a time resolution of 15 minutes.

Demand forecast is divided in electric demand and heating demand forecast, since high or low temperature heating can represent a considerable part of a building energy production. The tool is based on a spline regression algorithm, chosen in this case for its robustness. It is possible to write the effect of the load $y_{t}$ at instant $t$ as the sum of functions $f_{1}, \ldots, f_{k}$ associated with exogenous variables $x_{t}^{1}, \ldots, x_{t}^{K}$ and a random noise $\epsilon_{t}$

$$
y_{t}=\sum_{k=1}^{K} f_{k}\left(x_{t}^{k}\right)+\epsilon_{t} .
$$

With this approach, it is supposed that each exogenous variable influence the load independently. It is then necessary to find what the functions $f_{1}, \ldots, f_{K}$, approximated as splines. For this, historical data are used to train the model, i.e. different values of $x^{1}, \ldots, x^{K}$, and the result $y$ associated. A polynomial of the $3^{\text {rd }}$ order is then fitted in the vicinity of each point of this dataset. The complete function can then be obtained by adding the polynomial found at each point. This spline fitting is known to create smooth and relevant functions $f_{1}, \ldots, f_{K}$ with a reasonably large dataset and relatively good computing time.

- In the context of electric and heat demand load, three exogenous variables are crucial in the estimation of the load at time $t$ :

- The load of the day before, $\mathrm{y}_{\mathrm{t}-24}$, to capture the daily effect.

- The median load of the previous week, $\bar{y}_{t}$, to capture the trend of the current week. During long periods, electrical consumption is higher than during other.

- The temperature predicted for instant $t, \widehat{T}_{t}$, since outside temperature has a strong effect on electrical load.

Other exogenous variables such as cloud cover or week day are not used at the moment in order to do not increase the complexity of the model.

The PV production tool is similar in concept: Parameter's values are estimated depending on the time of day so as to capture interactions between the sun's course, the PV panels' orientation, potential shadowing effects, etc. It also allows capturing other effects from diurnal variations of meteorological parameters, such as the influence of temperature on modules' efficiency. Moreover the model's parameters are estimated adaptively using the most recent data available, so as to capture seasonal/climatic variations not explicitly modelled or even represented in the training data set (e.g. variations due to Numerical Weather Prediction (NWP) model's updates, to ageing or dirt on PV panels, etc.).

The chosen statistical model is a non-parametric model which has been considered in estimating the whole power distribution at once. It is based on a kernel density estimator (KDE) and can be written as:

$$
y_{t}=\frac{1}{n h} \sum_{i=1}^{n} K\left(\frac{x_{t}-x_{t, i}}{h}\right)
$$

Where $K$ is the kernel function and $h$ a smoothing parameter.

The exogenous variables for this model are:

- The historical production of the PV plant

- The predicted irradiance at instant $t$ for the location of the PV plant

The behavior of electrical flexibilities such as thermal energy storage (TES) and electric storage systems (ESS) units is managed at the high level layer by the Home Analytics tool. This tool has two main functions:

- Flexibility forecast

- Flexibility dispatch

The role of the flexibility forecast is to provide the EMSP with day-ahead estimations of the available electric demand flexibility and of its cost. This is provided for each independent user and for different level of aggregation such as a portfolio of clients or all the flexible loads in a certain network area. The cost of the flexibility is calculated considering the cost of the energy dissipated and the aging cost of the storage, particularly important in electrochemical ESS. The resulting cost function, in general non-linear, is linearized when sent to the EMSP. In total, the output of this forecast tool are:

- Maximum allowable power in charge [kW]

- Maximum allowable power in discharge $[\mathrm{kW}]$

- Maximum allowable energy in charge [kWh]

- Maximum allowable energy in discharge [kWh]

- Cost of discharge $[€ / \mathrm{kW}]$

- Cost of charge $[€ / \mathrm{kW}]$

A description of the architecture of the Flexibility forecast tool is presented in figure 4

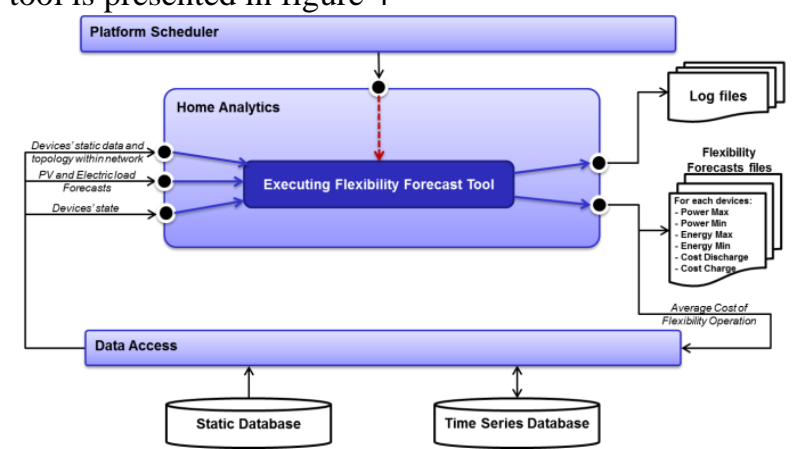

Figure 4: Block diagram showing the execution steps of the Flexibility Forecast Tool of the Home Analytics

The flexibility dispatch function, determines the set points of the devices that are capable of providing flexibility, according to the needs provided by the DSO or retailer. In order to do so, it considers as input: 1) the real time sate of charge of flexibility devices, 2) the real time electric demand and PV production, as they can limit the maximum flexibility and 3) the flexibility allocated at 
different aggregation level by the EMSP.

A description of the architecture of the Flexibility forecast tool is presented in figure 5 .

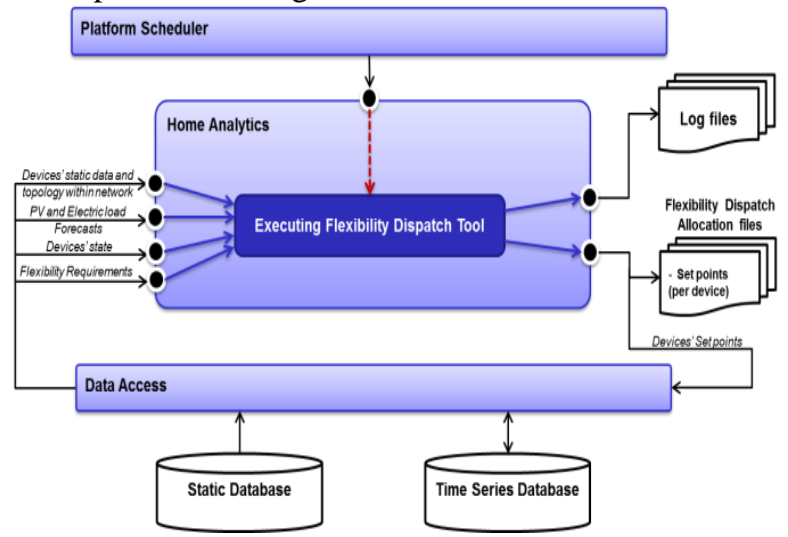

Figure 5: Block diagram showing the execution steps of the Flexibility Dispatch Tool of the Home Analytics

\section{VALIDATION AND NEXT STEPS}

Currently, tests have been realized for the PV production and for the electric demand forecast tools. The PV production forecast tool has been tested on the two PV plants currently in the area of the demo, for a period of roughly nine month between 2014 and 2015. It has been observed a normalized mean average error (NMAE) in the region between $10 \%$ and $11 \%$ of the rated power, a normalized root mean square error (NRMSE) between $14 \%$ and $15 \%$, as shown in fFigure 6.
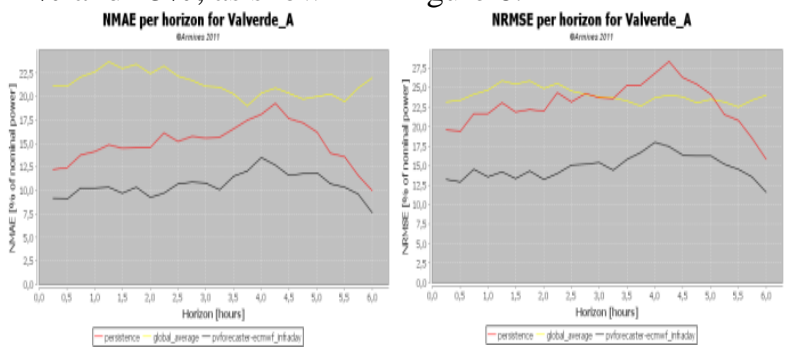

Figure 6: NMAE and NRMSE in different hours of the day for one producer in the demo area

The electric demand forecast tool has been tested on historical measurements of 226 households in the Evora area during 2015. It has been observed a symmetric mean absolute percentage error (SMAPE) in the region of $32 \%$ and an NMRSE in the region of $22 \%$, as shown in figureFigure 7

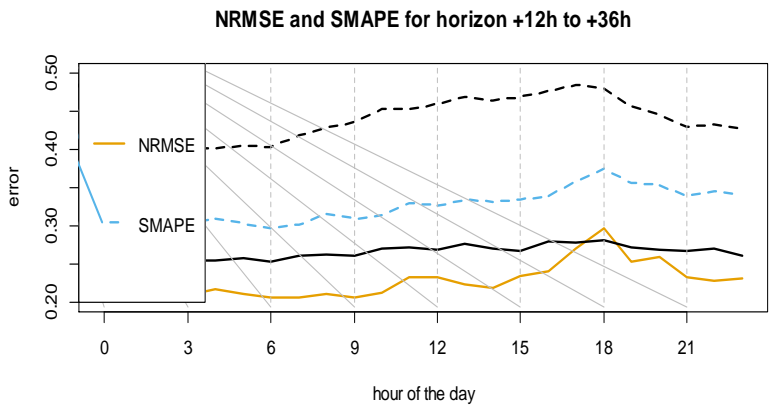

Figure 7: SMAPE and NRMSE in different hours of the day for all the consumers in the demo area

The figure 8 below illustrates the graphical calculations established in the EMSP. Flexibility requests from the DSO can include a desired capacity and also a price value. The platform processes these when comparing the DSO's and retailer's requests. On the other hand, DSO can also send flexibility requests without a price in an emergency situation. In these occasions the platform executes DSO's plans without a negotiation.

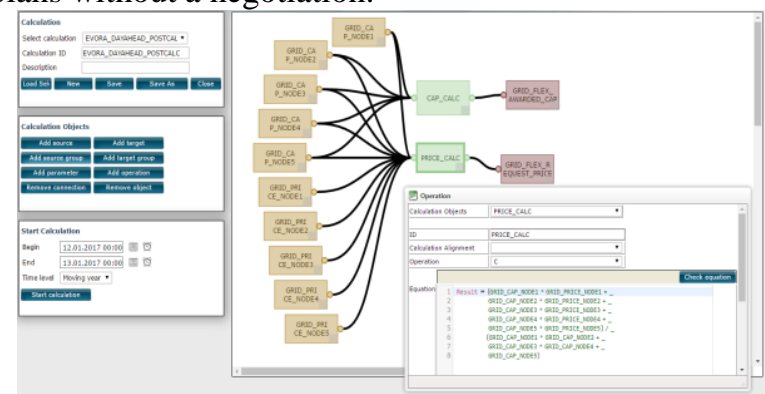

Figure 8 - Results from EMSP

At the moment the developments are in validation stage at lab environment and in next April the real demonstration in Valverde with real customers will start. This validation will be based in real market data and the actions will be made in these real customers in this real grid. An economic analysis is also ongoing in order to ratify this concept in full scale to retailers and energy services provides.

\section{ACKNOWLEDGMENT}

This work is being carried out as a part of the SENSIBLE project (Storage ENabled SustaInable energy for BuiLdings and communitiEs - www.h2020projectsensible.eu) that has received funding from the European Union's Horizon 2020 research and innovation programme under grant agreement No 645963.

\section{REFERENCES}

[1] - Gisela Mendes, Cired 2017, Community engagement in SENSIBLE project in both Évora and Nottingham demonstrators

[2] -Ricardo André, Cired 2016, customers' flexibility valued in market and regulated environment 